\title{
Cuatro momentos económicos en la historia del Perú Republicano'
}

\section{Four economical moments of the Republican history of Peru Jorge Américo Mendoza Vidal}

\section{RESUMEN}

En casi 200 años de historia republicana, hemos pasado por cuatro momentos económicos. El primero corresponde al boom del guano, de 1830 hasta 1876 . El segundo corresponde a la llamada República Aristocrática, período de 1894 a 1929. El tercero corresponde al boom exportador que vivimos luego del término de la Segunda Guerra Mundial, desde 1943 a 1976. El cuarto momento corresponde a los últimos 25 años de crecimiento económico mediante la aplicación de las políticas neoliberales. Se puede reconocer una tendencia en cada uno de estos.

A partir de los datos dispersos el articulo tiene como objetivo analizar los momentos económicos que permite reconocer tanto las fases de auge como de caída. Se recoge estadística actualizada de investigaciones e información de especialistas en economía para historiar estos cuatro momentos. La particularidad de estos momentos es que previamente a la configuración de cada uno se debatía políticas proteccionistas y de industrialización versus posiciones liberales de diferentes matices: primero, Proteccionismo frente a Libre Comercio; después se propone industrialización, se continua con Liberalismo: "laisser faire"; luego Keynesianismo frente a neoliberalis-

\footnotetext{
${ }^{1}$ Ponencia presentada en la Mesa de Historia Económica del VII Congreso Nacional de Historia celebrada en la Universidad Nacional de Trujillo (agosto - 2016).

${ }^{2}$ Jorge A. Mendoza es Historiador egresado de la Universidad Nacional Mayor de San Marcos. Miembro del Grupo de Investigación de Historia Económica y Social (ISHRA-UNMSM). Director de la revista de investigación Praxis en la Historia. Av. Guillermo de la Fuentes 612 Urbanización Santa Luzmila Comas, Lima, Perú.

(C) Los autores. Este artículo es publicado por Pensamiento Crítico de la Facultad de Ciencias Económicas, Universidad Nacional Mayor de San Marcos. Este es un artículo de acceso abierto, distribuido bajo los términos de la licencia Creative Commons Atribucion - No Comercia_Compartir Igual 4.0 Internacional. (http:// creativecommons.org/licenses/by-nc-sa/4.0/) que permite el uso no comercial, distribución y reproducción en cualquier medio, siempre que la obra original sea debidamente citada.
} 
mo. Podemos afirmar que la historia económica republicana se ha desarrollado en cuatro momentos económicos claramente definidos.

Palabras clave: Momentos económicos; Libre mercado; diversificación; crecimiento económico; debacle económica y conflictos sociales.

\section{ABSTRACT}

In almost two hundred years of republican history, we have passed for four economical moments. The first moment corresponds to the period of the "guano boom", from 1830 to 1876 . The second moment corresponds to the period of the called Aristocratic Republic, from 1894 to 1929 . The third moment corresponds to the "exportation boom" that we live after the end of the Second World War, from 1943 to 1976. The fourth moment corresponds to the lasts twenty five years of economic growth through the application of neoliberal policies. It can be recognized a trend in each of these moments.

Based on the scattered data it has been developed a diagram that permits recognize both phases, of boom and fall. It has been collected up-to-date statistics on research and information from economic specialists to write the history of these four moments. The particularity of these moments is that previously to the development of each one, there was always a debate on protectionist and industrialization policies against liberal positions from different nuances. First, Protectionism against Free Trade; then industrialization is proposed, continuing with Liberalism: "laisser faire"; then Keynesianism versus Neoliberalism. We can asseverate that the republican economic history has been developed in four economical moments clearly defined. Keywords: Economic moments; Free market; diversification; economic growth; economic debacle and social conflicts.

JEL: N16

\section{Introducción}

Hoy somos testigos que los 25 años de crecimiento económico que ha vivido el Perú desde los años 90, bajo las políticas neoliberales, ha terminado. Este crecimiento fue posible gracias a la venta de materias primas, es decir, un arrasamiento de nuestros recursos naturales y la concentración de lo producido por los trabajadores nacionales: artesanos, campesinos, obreros, técnicos y profesionales. Sin embargo, quienes se benefician con este crecimiento no es el pueblo peruano sino un pequeño grupo de propietarios de empresas multinacionales pertenecientes a las potencias imperialistas y grandes capitalistas que comandan este país para concentrar riquezas. 
Lo sintomático de estos últimos años está en la concentración de la riqueza que ha sido fríamente planificada desde los años noventa, como parte del 4. ${ }^{\circ}$ Plan Económico (Seminario 2011, p. 35), elaborado por los que durante casi 200 años de historia republicana vienen gobernando en nuestro país.

Este cuarto plan ya ha concluido, y no da para más. Ha repercutido aún más por las secuelas de la crisis del 2008, dejándonos en un momento económicamente crítico, en desaceleración con riesgo de recesión y, según algunos economistas, en camino al estancamiento si prosigue dentro de lo mismo como decía el economista Félix Jiménez en el 2015, y según Gianfranco Castagnola, Presidente Ejecutivo de Apoyo Consultoría, quien a mediados del 2017 señaló que "Hay un estancamiento con sensación de recesión".

Por ello, ante las secuelas de la crisis del 2008 y el camino a un nuevo reparto del mundo evidenciándose más los frentes militares y la antesala de una guerra mundial imperialista, las organizaciones al servicio de las potencias mundiales como el Fondo Monetario Internacional (FMI) y el Banco Mundial (BM) vienen recomendando la necesidad de activar las políticas fiscales y las reformas adecuadas para incentivar mayor gasto público; los críticos del neoliberalismo y defensores del sistema capitalista proponen centrar en impulsar el consumo interno y elevar la productividad a la vez que los salarios. Sin embargo, estas recomendaciones en nuestro país aún no han sido definidas, por lo cual sostenemos llevaría a los que dirigen el país a la elaboración de un 5 . $^{\circ}$ plan económico, pero los grandes capitalistas aun no tienen consenso en cómo van a implementar las recomendaciones económicas que permitan una "nueva normalidad" más estable, es un tema que vienen postergando y que tendrán que definir en el Perú, antes del Bicentenario.

Lo que se evidencia claramente es que ha terminado un momento del proceso económico de la sociedad peruana y debe definirse otro, el cual abarcaría un período de 30 a 35 años aproximadamente. Es un serio e importantísimo problema para el Perú, es un tema de los que mandan en este país.

El llamado Plan de Diversificación Productiva (Ministerio de la Producción 2014) consideramos que fue parte de lo que se estuvo proponiendo como plan quinto o quinto momento, pero este tomaría 
entre 10 a 15 años implementarlo así como una gran inversión, por lo que es aún insuficiente e incompleto y el actual gobierno de PPK al parecer lo ha sacado de sus prioridades. De todas maneras habrá nuevo plan y los grandes empresarios lo harán incluso urgidos por el bicentenario para el cual les falta 5 años todavía.

De ahí, el interés de conocer y comprender los momentos económicos por los cuales nuestro país ha pasado en estos dos siglos de existencia, cómo se han dado las contiendas entre facciones de grupos de poder y las luchas del pueblo peruano que del peso de la explotación surgen en estos proceso económicos.

\section{Estudios que toman como base los momentos económicos}

Los estudios de historia económica en nuestro país conciben los cuatro momentos a partir de los estudios realizados por Rosemary Thorp y Geoffrey Bertram en los años 70s que llevó a la publicación de Perú: 1890 - 1977. Crecimiento y política en una economía abierta. Estos autores sustentan que la historia del comportamiento de las exportaciones está dividida en tres fases distintas de aproximadamente cincuenta años de duración cada una. Que los periodos de mayor estabilidad política y de conservadurismo en la historia peruana - la "república aristocrática" y el "oncenio" de 1895 a 1930, y los regímenes de Odría y Prado de 1948 a 1962corresponden a extensos periodos de auge de la economía de exportación, mientras que los años de inestabilidad - de 1882-1895, de 1930 a 1948 y finales de la década 1960 correspondieron a los periodos en los que la economía de exportación ingresó a un estado de crisis y dejó de producir claros lineamientos de política económica; por ello es considerado "el estudio más importante de la economía peruana desde la perspectiva de las relaciones internacionales (...). La disponibilidad de recursos naturales valiosos para el mercado mundial, la institucionalidad que los extrae y comercia, y los efectos de la exportación sobre el proceso económico interno, son materia de pormenorizado análisis". (Escobal e Iguíñiz 2000, p. 138). 
Este estudio fue un documento que permitió una visión estratégica del Perú y que historiadores, economistas, políticos y científicos sociales de otras ciencias han tomado para sustentar el marco histórico de sus investigaciones ${ }^{3}$. El caso más representativo ha sido el realizado por Bruno Seminario, Cynthia Sanborn y Nikolai Alva quienes ante la necesidad de ir respondiendo sobre el rumbo económico que nuestro país tendrá en los próximos 30 a 35 años realizaron importantes investigaciones desde el Centro de Investigaciones de la Universidad del Pacifico que concluyeron entre las más importantes en su libro Cuando despertemos en el 2062, una pregunta que en su repuesta presenta una visión de 50 años, en la que se señala que las depresiones de la economía peruana presentan una duración de aproximadamente 17 años, y las fases de auge, con tasas de crecimiento no muy elevadas, duraciones promedio de 35 años. Estas fases de auge y caída configurarían, por su reiteración, una suerte de ciclo económico representativo de la economía peruana de aproximadamente 51 años de duración.

Quienes usan como marco histórico el trabajo de Rosemary Thorp y Geoffrey Bertram para la formulación de una propuesta de un quinto plan económico en el país, son los economistas José Gallardo y Piero Ghezzi en su libro titulado también con una interrogante a futuro ¿Qué se puede hacer con el Perú? (2013). El marco histórico de este libro también se basa en la existencia de cuatro períodos de la economía peruana de altos niveles de crecimiento por largos períodos de tiempo para luego caer en largos períodos de recesión. El primero corresponde al boom del guano, de 1830 hasta 1876, antes de la guerra con Chile, cuando el Perú creció a una tasa promedio de 3.4\%. El segundo momento corresponde a la llamada República Aristocrática y el Oncenio de Leguía, período de 1894 a 1929 cuando el PBI se incrementó a un ritmo de 5.1\% anual. La tercera corresponde al boom exportador que vivimos luego del término

\footnotetext{
${ }^{3}$ Claro ejemplo de como la obra de Rosemary Thorp y Geoffrey Bertram sirve como marco histórico se refleja en los trabajos presentados en el Tomo 4 del Compendio de Historia Económica del Perú editado por Carlos Contreras Carranza y auspiciado por el BCRP y el IEP, donde los historiadores Fernando Armas Asín, José R. Deustua C., Martín Monsalve y Alejandro Salinas lo tienen en su referencias, haciéndose en este Compendio una mención especial en el "Apéndice cuantitativo: El Perú entre 18211930" por Luis Miguel Espinoza.
} 
de la Segunda Guerra Mundial, ocasión en que se creció a razón de 5.3\% anual desde 1943 a 1976.

\section{Cuatro momentos económicos en el Perú republicano}

Desde comienzos de la República (1821), se han desenvuelto cuatro grandes momentos en el proceso del crecimiento económico peruano. El primero de 1830 a 1876, centrado en el "boom" del guano; el segundo de 1894 a 1929 centrado en minería y agroproducción, el "boom” del azúcar; el tercero de 1943 a 1976 asociado al "laisser faire", es decir libre mercado, con un mayor impulso de la minería, la pesca también; y el cuarto, el del neoliberalismo centrado en el "boom" de los altos precios de los minerales y las exportaciones, que viene desde 1990 pero que ya habría concluido.

\section{Cuadro 1.}

Cuatro momentos económicos en la historia del Perú republicano

\begin{tabular}{|l|l|l|l|l|}
\hline MOMENTOS & $\begin{array}{l}\text { PRIMER } \\
\text { MOMENTO }\end{array}$ & $\begin{array}{l}\text { SEGUNDO } \\
\text { MOMENTO }\end{array}$ & $\begin{array}{l}\text { TERCER } \\
\text { MOMENTO }\end{array}$ & $\begin{array}{l}\text { CUARTO } \\
\text { MOMENTO }\end{array}$ \\
\hline PERÍODO & $1830-1876$ & $1894-1929$ & $1943-1976$ & $1990-2011$ \\
\hline $\begin{array}{l}\text { BOOM } \\
\text { ECONÓMICO } \\
\text { (duran aprox. } \\
\text { 30 a 35 años) }\end{array}$ & Guano & Azúcar & Minería y pesca & Minería \\
\hline PBI promedio & $3.4 \%$. & 5.1\% & 5.3\% & 5.4\% \\
\hline $\begin{array}{l}\text { DEBACLE } \\
\text { ECONÓMICo }\end{array}$ & $\begin{array}{l}\text { Guerra del Pacifico } \\
\text { y su repercusión } \\
(1877-1893)\end{array}$ & $\begin{array}{l}\text { Repercusiones } \\
\text { Crack del 29 } \\
(1930-1943)\end{array}$ & $\begin{array}{l}\text { Crisis de precios } \\
\text { del 73 } \\
(1976-1990)\end{array}$ & $\begin{array}{l}\text { Desaceleración } \\
\text { económica con } \\
\text { riesgo de recesión }\end{array}$ \\
\hline $\begin{array}{l}\text { DEBATE } \\
\text { (AI Concluir el } \\
\text { periodo) }\end{array}$ & $\begin{array}{l}\text { Proteccionismo } \\
\text { frente a Libre } \\
\text { comercio }\end{array}$ & $\begin{array}{l}\text { Proponen } \\
\text { industrialización, } \\
\text { se continua con } \\
\text { Liberalismo: } \\
\text { "laisser faire" }\end{array}$ & $\begin{array}{l}\text { Keynesianismo } \\
\text { frente a } \\
\text { neoliberalismo }\end{array}$ & $\begin{array}{l}\text { Diversificación } \\
\text { Productiva: para } \\
\text { mercado interno o } \\
\text { para exportación }\end{array}$ \\
\hline
\end{tabular}

Elaboración: Propia a partir de las investigaciones de Thorp y Bertram (1985), Seminario, Sanborn y Alva (2012), Gallardo y Ghezzi (2013)

José R. Deustua C., Martín Monsalve y Alejandro Salinas lo tienen en su referencias, haciéndose en este Compendio una mención especial en el "Apéndice cuantitativo: El Perú entre 1821-1930" por Luis Miguel Espinoza.

El cuadro elaborado nos permite analizar estos cuatro momentos con más detalle. Fijémonos, por ejemplo, en los periodos de mayor auge económico de exportaciones en los que se han vivido momentos de 
relativa estabilidad política con la presencia de gobiernos populistas como el Oncenio de Leguía del periodo 1894 a 1929 o el Ochenio de Odría y la dictadura de Velasco Alvarado del periodo de 1943 a 1976 y la llamada dictadura cívico-militar de Alberto Fujimori del último periodo.

\section{Subidas y caídas de nuestra economía}

Los momentos de debacle económica, producto de la baja de las exportaciones, han sido momentos en los que se han agudizado más los conflictos políticos en nuestro país, como lo señalan Thorp y Bertram: "No sorprende descubrir que los periodos de mayor estabilidad política y de conservadurismo en la historia peruana - la "república aristocrática" y el "oncenio" de 1895 a 1930, y los regímenes de Odría y Prado de 1948 a 1962- corresponden a extensos periodos de auge de la economía de exportación, mientras que los años de inestabilidad - de 1882-1895, de 1930 a 1948 y finales de la década 1960 correspondieron a los periodos en los que la economía de exportación ingresó a un estado de crisis y dejó de producir claros lineamientos de política económica.(1985, p. 6-7)"

Esto ha sucedido en los cuatro momentos económicos por los que ha pasado nuestra historia republicana, como se dio al final del primer momento económico con la Guerra de Chile contra Perú y Bolivia, y la Guerra Civil entre el Mariscal Avelino Cáceres y Nicolás de Piérola en la debacle económica de 1877 a 1893; así también finalizado el segundo momento económico después del Crack del 29 se dio un dura persecución política con Sánchez Cerro y Oscar Benavides; finalizado el tercer momento se dio duras luchas del pueblo peruano por una Asamblea Constituyente y poco después en los 80 se inició el conflicto armado interno de mayor trascendencia de nuestra historia. Ahora, terminado este cuarto momento, ya vemos como luchas masivas se oponen a la explotación capitalista e imperialista agudizándose cada vez más la contradicción pueblo-capitalismo.

Los trabajos de Bruno Seminario (2011) muestran el seguimiento cada vez más detallado de estos momentos económicos, de sus auges y descensos. 
Cuadro 2.

Fases de auge y descenso del PBI peruano

\begin{tabular}{|c|c|c|c|c|c|c|c|c|}
\hline & & & \multicolumn{3}{|c|}{ Periodos } & \multicolumn{3}{|c|}{ Valores } \\
\hline & & & $\begin{array}{l}\text { Año de } \\
\text { Inicio }\end{array}$ & $\begin{array}{l}\text { Año de } \\
\text { Fin }\end{array}$ & Duración & $\begin{array}{c}\text { Valor de } \\
\text { Inicio } \\
\end{array}$ & $\begin{array}{c}\text { Valor de } \\
\text { Fin }\end{array}$ & $\begin{array}{c}\text { Crecimiento } \\
\text { promedio }\end{array}$ \\
\hline & B & Downswing & 1706 & 1723 & 17 & 429822 & 311098 & $-1.58 \%$ \\
\hline \multirow{2}{*}{ I } & A & Upswing & 1723 & 1747 & 24 & 311098 & 468100 & $1.61 \%$ \\
\hline & $\mathrm{B}$ & Downswing & 1747 & 1755 & 8 & 468100 & 458424 & $-0.26 \%$ \\
\hline \multirow{2}{*}{ II } & A & Upswing & 1755 & 1795 & 40 & 458424 & 873199 & $1.61 \%$ \\
\hline & $\mathrm{B}$ & Downswing & 1795 & 1830 & 35 & 873199 & 636362 & $-0.90 \%$ \\
\hline \multirow{2}{*}{ III } & A & Upswing & 1830 & 1876 & 46 & 636362 & 3032961 & $3.37 \%$ \\
\hline & $\mathrm{B}$ & Downswing & 1876 & 1894 & 18 & 3032961 & 1621164 & $-3.48 \%$ \\
\hline \multirow{2}{*}{ IV } & A & Upswing & 1894 & 1929 & 35 & 1621164 & 10292401 & $5.28 \%$ \\
\hline & $\mathrm{B}$ & Downswing & 1929 & 1943 & 14 & 10292401 & 12224875 & $1.23 \%$ \\
\hline \multirow{3}{*}{ V } & A & Upswing & 1943 & 1976 & 33 & 12224875 & 66506572 & $5.13 \%$ \\
\hline & $\mathrm{B}$ & Downswing & 1976 & 1992 & 16 & 66506572 & 66130062 & $-0.04 \%$ \\
\hline & $\mathrm{A}$ & Upswing & 1992 & ¿2027? & - & 66130062 & - & - \\
\hline
\end{tabular}

Los valores del PIB están expresados en términos reales en miles de \$ Geary-Khamis. Se construyó sobre la data histórica 1700-2010, reconstruida en el presente documento.

Fuente: Luis Bruno Seminario De Marzi: "Las Cuentas Nacionales del Perú, 1700-2010"

Este cuadro muestra, con mayor detalle, los tiempos de auge y los tiempos de descenso económico, en el que claramente se presenta cada momento histórico, incluidos aquellos que se dieron antes de nuestra constitución como República. Refleja nítidamente el promedio general entre auges y descensos que tienen una duración de 50 años aproximadamente, entre periodos de expansión de 35 años y de contracción de 17 años; donde la recomendación es evaluar el potencial de la economía peruana diferenciando estas dos fases que son una constante en nuestro proceso económico.

\section{Cuadro 3.}

Promedios y desviaciones estándar de las fases de auge y desarrollo del PBI Peruano

\begin{tabular}{lcr} 
& $\begin{array}{c}\text { Años de } \\
\text { Duración }\end{array}$ & $\begin{array}{c}\text { Tasas de } \\
\text { Variación }\end{array}$ \\
\hline Promedio de Upswing & 34.60 & $3.46 \%$ \\
Promedio de Downswing & 17.17 & $-0.99 \%$ \\
Mediana de Upswing & 34.00 & $3.38 \%$ \\
Mediana de Downswing & 16.00 & $-0.01 \%$ \\
Desviación estándar Upswing & 8.20 & 0.0477 \\
Desviación estándar Downswing & 9.04 & 0.0669 \\
\hline
\end{tabular}

Fuente: Luis Bruno Seminario De Marzi: “Las Cuentas Nacionales del Perú, 1700-2010” 


\section{Los debates y definiciones: formas de libre mercado}

Al finalizar cada periodo, en medio del debacle económico se dieron debates para definir el rumbo a seguir, obviamente eran discusiones entre los que tienen el poder, para definir de qué forma se iba a explotar al pueblo las siguientes décadas. Existía incertidumbre sobre la viabilidad del crecimiento de las exportaciones en el futuro y se debatía cual era el papel que debía cumplir el gobierno en la economía, algo así como los debates que hoy se hacen por la competitividad y el fortalecimiento de las instituciones del Estado.

En los años del primer y segundo auge, se habría dado un debate muy arduo entre el problema del proteccionismo y el libre mercado, definiéndose por lo último. En los años 30 se dio un interés por promover la industrialización para salir de la caída, sin embargo, hubieron posiciones de economistas liberales que plantearon el "laisser faire", expresión francesa que significa "dejen hacer, dejen pasar», la cual se impuso finalmente. Terminado el tercer periodo se dio un descenso en momentos en los que se experimentó por primera vez con el neoliberalismo en Chile y en que se quería dar más protagonismo en el Perú al sector privado frente al control estatal, imponiéndose finalmente, como en todo el mundo, el neoliberalismo que vino acompañada de una política de arrasamiento de los derechos fundamentales del pueblo. En el esquema que a continuación presentamos se puede visualizar mejor las posiciones enfrentadas en cada momento económico:

1890: Luego de la Guerra del Pacífico se exploraron nuevas estrategias para el desarrollo y se debatió arduamente el problema del proteccionismo frente al libre comercio.

1948: La gran depresión y la Segunda Guerra Mundial se reflejaron en cierto desconcierto respecto del crecimiento por expansión de las exportaciones y en un nuevo interés por la industrialización como posible estrategia a seguirse. 1940: al no darse un nuevo crecimiento en las exportaciones, el Perú entró a una corta era de controles económicos y de racionamiento antes de regresar en 1948 a las tradicionales políticas de liberalismo. 
1973: Cuando la economía de exportación afrontó dificultades una junta militar distinta a la facción velasquista tomó el poder e implementó una serie de medidas económicas de tipo radicalnacionalistas (Thorp y Bertram1985, 7). Para luego del término del proceso armado interno y del golpe de estado de Fujimori imponerse un camino neoliberal.

2015: Repercutido con la última Crisis Mundial del 2008, la más grande vivida en el capitalismo y la segunda de la globalización, el Perú concluyó actualmente sus 25 años de crecimiento económico, desenvolviéndose en una desaceleración económica con riesgo de recesión y camino al estancamiento. Esto ha llevado a definir un nuevo rumbo económico y un quinto plan que el actual gobierno de PPK tendrá que aplicar.

Por último, ya finalizado el cuarto momento de nuestra economía, a diferencia de los anteriores momentos, se carece de una ideología base de una nueva política económica y todavía no se define el cómo desarrollar un nuevo plan económico a futuro; lo que sí está presente es el término de un momento y la necesidad de definir otro.

\section{Camino a un quinto momento económico}

El Perú, en los últimos diez años, ha sido visitado por reconocidos economistas neoliberales de formación norteamericana en la Universidad de Harvard que han puesto en alerta a los grandes empresarios capitalistas peruanos sobre el fin del crecimiento económico dentro del neoliberalismo y la necesidad de impulsar un nuevo plan a seguir.

El primero en llegar (2009) fue Michael Porter ${ }^{4}$, quien fuese presentado como "el gurú mundial sobre estrategia y competitividad". Realizó observaciones, que la prensa peruana las calificó de duras, a la política económica peruana, entre ellas señaló que nuestra economía "no tiene un rumbo definido" (Correo, 12.12.09), en clara alusión al que el proceso económico neoliberal ya no podía seguir siendo su guía. Señaló que "el Perú ha vivido en estos años una ilusión exportadora (...) mientras que la exportación de productos con valor agregado permanece inmóvil”

\footnotetext{
${ }^{4}$ Fue invitado a un evento organizado por la Universidad del Pacífico e Interbankrealizado el mes de noviembre del 2009, titulado: "Seminario Internacional: Claves de una Estrategia Competitiva".
} 
(La Primera, 03.12.09), inyectando así la ilusión de la diversificación de la producción. Por último, dijo que "los éxitos peruanos de los últimos años pueden irse al demonio sino limpiamos el sistema judicial, sino defendemos los derechos de propiedad y si no fumigamos y reordenamos la disuasiva burocracia creada para entorpecer"(La Primera, 03.12.09), medidas que van acorde a lo señalado por los supuestos economistas "críticos" del neoliberalismo que proponen centrar en impulsar el consumo interno y elevar la productividad a la vez que reformar las instituciones.

A mediados del 2013, llego a nuestro país Ricardo Hausmann ${ }^{5}$ quien planteó que "el Perú debe diversificar su economía para acelerar su crecimiento" (El Comercio, 17.04.13), haciendo más énfasis en la necesidad de diversificar la producción: "el Perú enfrenta un tipo de cambio menos competitivo, lo cual en muchos casos no ha permitido la diversificación de las exportaciones"(El Comercio, 17.04.13). Obviamente orientan a generar explotación capitalista en otras ramas de la producción, dice Hausmann "Está claro que el camino del Perú es la diversificación, porque permite usar toda la capacidad productiva de la gente"(Gestión,11.06.13), claramente apunta a la plusvalía, pues qué otra cosa puede resultar de la capacidad productiva de la gente.

Por último, el 2014 llegó Dani Rodrik ${ }^{6}$ con una noticia negativa para la política económica del país, señaló que "el crecimiento de los países en desarrollo o emergentes no se podía sostener a los niveles que habíamos visto en la última década" (Peru21, 23.01.14). Recalcó que el 2013 fue el año en el que "la burbuja de mercados emergentes se reventó para siempre"(Peru21, 23.01.14). A la gran burguesía, después de vender el cuento de que estábamos blindados, le estalló en la cara las secuelas de la crisis del 2008.

Analizando las opiniones vertidas por estos especialistas,que hablan de la situación económica del país, consideramos que los que mandan en el Perú no tienen definido su rumbo económico, es por ello que el imperialismo norteamericano envío a sus principales economistas de la

\footnotetext{
${ }^{5}$ Economista venezolano director del Centro para el Desarrollo Internacional de la Universidad de Harvard estuvo en Lima el 2013 para participar en el foro económico organizado por la Cámara de Comercio de Lima y en las actividades por el 117 aniversario de la Sociedad Nacional de Industrias.

${ }^{6}$ Economista formado en Princeton y Harvard especializado en temas de desarrollo económico y reformas de Estado fue entrevistado por Peru21 en el contexto del Foro Económico Mundial del 2014.
} 
Universidad de Harvard para ir orientando la economía peruana al ritmo de las necesidades políticas y económicas de EE. UU.

Ahora les urge que el actual Gobierno de PPK zanje con el rumbo económico a seguir de ahí que hoy se use la reconciliación nacional, para como dice Mercedes Araoz "continuar y, sobre todo, profundizar las reformas en favor de todos nuestro compatriotas" (El Comercio, 07.01.18). No tienen definido un plan económico cada facción política de la gran burguesía quiere beneficiarse económicamente y hoy usan la necesidad de una verdadera reconciliación nacional como componenda política económica.

\section{Debate sobre el rumbo a seguir}

Hemos querido mostrar algunas posiciones que existen sobre el rumbo a seguir ahora que ha terminado el cuarto momento económico, debate que protagonizan diferentes instituciones y economistas, así como autoridades gubernamentales.

Una de las instituciones que se ha pronunciado sobre el tema es la Sociedad Nacional de Minería Petróleo y Energía (SNMPE) en la que su presidente Carlos Gálvez señala: "Con estabilidad jurídica se pueden retomar las inversiones", "respeto a los convenios de estabilidad jurídica y tributaria", "reforma de todo el sistema legal que se ha manejado en los últimos años" (Correo: 29.02.2016). "Institucionalidad", porque "hay un deterioro institucional" y "pésima eficiencia del Estado". La SNM plantea sus posiciones centrando en generar las bases jurídicas, tributarias y sociales para ir preparando las condiciones que les permita una forma diferente de concentración de capitales.

Eduardo Jiménez, uno los mayores defensores de la gran minería, tiene posiciones similares a la SNM cuando señala que: "En principio, no hay ningún riesgo de que el modelo económico que ha hecho crecer al país en los últimos 20 años sea modificado, pero la dosis de populismo cada vez se hace más evidente. Por ejemplo, los principales candidatos ofrecen, de una u otra manera reducir los impuestos cual si fuera la solución mágica a todos los problemas de la informalidad en el país. Ante ello hay que indicar que el país necesita elevar su gasto público en educación y salud y para ello se necesita seguir elevando la presión tributaria". (Gestión: 
30.12.2015). Eduardo Jiménez pone el peso en la concentración tributaria convergiendo con la SNM para beneficiar sus ganancias, pero también plantea la necesidad de una estabilidad que permita seguir concentrando mediante reformas que mantengan el modelo neoliberal como fondo.

De igual forma y en la misma línea de solución Luis Carranza, ex ministro de Economía y Finanzas, reflexiona algunas medidas: "Ojalá el siguiente gobierno tenga la capacidad de priorizar el gasto público, tomando la inversión privada como la principal aliada para el desarrollo del país y finalmente, que tenga la capacidad de seleccionar bien y llevar adelante las reformas que el país necesita". (El Comercio: 19.01.2016). En esencia los tres anteriores representantes de las posiciones de la Gran Burguesía señalan reformas e intervención estatal mediante políticas públicas pero sin cambiar el modelo económico de fondo que es el neoliberal, lo único que buscan es remozar el sistema con políticas neoliberales de "segunda generación".

Por eso el economista Eduardo Jiménez más abiertamente concretiza la propuesta específica para el rumbo a seguir: "En el actual entorno en el que la inversión privada se encuentra cayendo es necesario que el sector público la apoye vía asociaciones público-privadas o iniciativas privadas. De esta manera iremos regresando, aunque lentamente, a nuestro crecimiento potencial de 4.5 a 5\%”. (Gestión: 20.01.2016). Posiciones que socialmente hablando terminan siendo recalcitrantes, pues hunden más las desigualdades buscando beneficiar y salvar sus empresas e inversiones y a la larga abandonar a la población quienes serán al final quienes cargarán el peso de la crisis con las reformas tributarias, laborales y sociales que lleva a privatizar la educación y la salud.

Ahora, diferenciándose de las posiciones recalcitrantes antes presentadas hay posiciones más avanzadas que se expresaron en "Presente y futuro después del 2015" realizada por diario UNO el 31 de diciembre del 2015, donde, entre otras cosas, propone: "alejarse de la excesiva dependencia de materias primas lo que se extiende a una dependencia de China, EEUU y Europa", "lograr la diversificación productiva" y que según Fernando Villarán, entre otros economistas entrevistados, señaló que "el Estado timonee la industrialización como los países de la segunda ola industrializadora Canadá, Australia, Corea del Sur". Estas posiciones 
plantean una industrialización pero al no tener un plan económico ni organizaciones sociales que enrumben a ese camino quedan solo en ideas.

Cabe destacar la propuesta de Félix Jiménez quien muy crítico del neoliberalismo propone "cambiar el estilo de crecimiento": "...La actual crítica situación económica en el contexto de una profunda crisis política ha generado nuevamente una gran oportunidad para iniciar el camino al desarrollo. Es la hora de cambiar el modelo de crecimiento y acumulación del capital. Es la hora de emprender un proceso de industrialización de nuevo tipo basado en la agricultura y en la agroindustria", "se abre la posibilidad de un manejo soberano de la política monetaria... y de cambio en la orientación de la política fiscal y de inversiones públicas", "basar el financiamiento del crecimiento en el desarrollo del mercado de capitales en moneda local". (Diario UNO: 19.12.2015). Estas posiciones expresadas en función a una industrialización es una propuesta realmente necesaria, pero en las condiciones políticas en las que se encuentra el país se hace poco viable, más aun al no contar con un plan económico definido.

Lo que actualmente existe es un debate al igual que en los momentos económicos anteriores pero todavía no se define el cómo aunque sí está presente el término de un momento y la necesidad de definir otro. Igualmente se manifiesta también la contradicción pueblo-capitalismo, pues como siempre el pueblo y la última clase de la historia pagarán el costo; se incrementará la explotación para obtener más ganancias y mayor capital, así como igualmente en esta perspectiva se impulsará la apropiación de medios productivos. De modo que se presenta la necesidad una vez más de dar rumbo desenmascarando y denunciando al gran capital pro imperialista o imperialista, a sus clases sociales la oligarquía financiera y las facciones de la gran burguesía, así como sus planes y perspectivas.

\section{Conclusión}

Estamos próximos a cumplir 200 años como República y esto nos lleva a ver al Perú en su economía y su historia, en un proceso largo que permita comprender su dinámica política y social y nos lleve a desarrollar nuestra nación. Para ello presentamos las conclusiones en tres puntos que bosquejan una problemática mayor: 
Primero: se debe tener en cuenta que el Perú ha pasado por cuatro momentos económicos muy influenciados por la situación económica internacional; tanto en las etapas de crecimiento como de recesión, durando un promedio de 50 años. Los crecimientos económicos hasta el final del auge duraron un promedio de 30 a 35 años y las etapas de caída y repercusiones negativas duraron un promedio de 15 a 17 años.

Segundo: las crisis mundiales han marcado hitos para cada momento económico en el Perú y han devenido en conflictos sociales profundos. La crisis de Europa en los años 70 del siglo XIX no solo acabó con la explotación del guano, además repercutió para que se desarrolle la Guerra del Pacifico y la posterior Guerra Civil entre Cáceres y Piérola. El histórico Crack del 29 terminó con el boom del azúcar y el algodón y además repercutió muy duro socialmente generándose como respuesta la persecución política a comunistas y Apristas por parte de la Unión Revolucionaria encabezada por Sánchez Cerro. El año 1973 con la famosa crisis de los precios termina el boom económico de harina de pescado y minerales, así también concluye la etapa de la Comisión Económica para América Latina y el Caribe (CEPAL) y se inicia la aplicación del neoliberalismo, desarrollándose una protesta social enorme llevando a la Constituyente del 79 y al inicio de una nueva Guerra Civil en los años 80s. Ahora con la crisis del 2008 se da fin al boom de los minerales y se vienen dando luchas masivas nunca antes vistas en nuestro país.

Tercero: se hace necesario para los historiadores económicos desarrollar una investigación de gran magnitud en la que se pueda vislumbrar los cuatro momentos económicos en la historia de nuestra República, para comprender cómo estamos dentro de un círculo vicioso que no lleva a buen puerto a nuestro país. Permitiéndonos como alternativa retomar los planteamientos de políticas económicas de desarrollos autónomos.

Finalmente la gran burguesía o, para algunos, los grandes empresarios, es decir los que mandan en el país, tienen claro estos momentos económicos y pretenden contrarrestar la caída y repercusión de la crisis, sin embargo, hoy es inminente una recesión y por ello estamos históricamente advertidos que los conflictos sociales se van a agudizar y el enfrentamiento entre peruanos corre el riesgo de no tener solución. Por eso, es necesario entrar a este Bicentenario solucionando problemas no resueltos que permitan el desarrollo mayor de nuestra nación. 


\section{Referencias bibliográficas}

Aráoz Mercedes (2018). “Diálogo y reconciliación para la prosperidad. El Comercio: 07 de enero.

Carranza Luis (2016). "No más mediocridad". El Comercio: 19 de enero.

CastagnolaGianfranco (2017). Entrevista. El Comercio.25 de junio.

Dani Rodrik, (2014). “Entrevista” Peru21: 23 de enero

Escobal Javier e Iguíñiz Javier (2000).Balance de la Investigación Económica en el Perú. Lima: Consorcio de Investigación Económica y Social.

El Comercio (2013): "Hausmann: El Perú debe diversificar su economía para acelerar su crecimiento". Lima: 17 de abril

Gallardo José y Ghezzi Piero(2013). Qué se puede hacer con el Perú: ideas para sostener el crecimiento económico en el largo plazo.1 edición. Lima: Universidad del Pacífico y Pontificia Universidad Católica del Perú.

Gálvez Carlos (2016). Entrevista. Correo: 29 de febrero.

Hausmann Ricardo (2013). “Entrevista”. Gestión: 11 de junio.

Hildebrandt Cesar (2009). "Estupefactos". La Primera. 03 de diciembre.

Jimenez Eduardo (2015). “2016, un año lleno de retos”. Gestión: 30 de diciembre. (2016) “China, principal riesgo en 2016”. Gestión: 20 de enero.

Jiménez Félix (2015). “Cómo salir del estancamiento económico”. Diario Uno. 19 de diciembre.

Lago V. Ricardo (2009). “Los cuentos de Porter”. Correo:12 de diciembre.

Ministerio de la Producción (2014). Plan Nacional de Diversificación Productiva. Lima: Minpro - PRODUCE.

Rosemary Thorp, y BertramGeoffrey (1985). Perú, 1890-1977. Crecimiento y políticas en una economía abierta. Lima: Universidad del Pacífico, Fundación F. Ebert y Mosca Azul.

Seminario De Marzi, Luis Bruno (2011). "Las Cuentas Nacionales del Perú, 17002010” (Estimados Preliminares). DOCUMENTO DE DISCUSIÓN DD/12/11. LIma: Universidad del Pacifico - Centro de Investigación.

Seminario Bruno, Sanborn Cynthia y Alva Nikolai (2012) Cuando despertemos en el 2062, visión del Perú en 50 años.1ae edición.Lima: Universidad del Pacífico. 